\title{
AGRONYMS AND ABBREVIATIONS
}

$\begin{array}{ll}\text { ABRI } & \text { Angkatan Bersenjata Republik Indonesia } \\ \text { ACC } & \text { Appointments Committee of the Cabinet } \\ \text { ACSA } & \text { Acquisition and Cross-Servicing Agreement } \\ \text { AFC } & \text { Allied Forces Command } \\ \text { AFD } & \text { Armed Forces Division } \\ \text { AFP } & \text { Armed Forces of the Philippines } \\ \text { AFPFL } & \text { Anti-Fascist People's Freedom League } \\ \text { AMC } & \text { Army Medical Corps } \\ \text { ANSP } & \text { Agency for National Security Planning } \\ \text { ASC } & \text { Army Security Command } \\ \text { ASEAN } & \text { Association of Southeast Asian Nations } \\ \text { AWT } & \text { Army Welfare Trust } \\ \text { Bayan } & \text { Bagong Alyansang Makabayan } \\ \text { BJP } & \text { Bharatiya Janata Party } \\ \text { BNP } & \text { Bangladesh Nationalist Party } \\ \text { BSF } & \text { Border Security Force } \\ \text { BSPP } & \text { Burma Socialist Program Party } \\ \text { Bulog } & \text { National Logistics Board } \\ \text { CAO } & \text { Civil Affairs Office } \\ \text { CBCP } & \text { Catholic Bishops Conference of the Philippines } \\ \text { CCP } & \text { Chinese Communist Party } \\ \text { CCPA } & \text { Cabinet Committee on Political Affairs } \\ \text { CDF } & \text { Ceylon Defense Force } \\ \text { CDNS } & \text { Council for Defense and National Security } \\ \text { CENTO } & \text { Central Treaty Organizaton } \\ \text { CGS } & \text { chief of the general staff } \\ \text { CHDF } & \text { Civilian Home Defense Forces } \\ \text { CHT } & \text { Chittagong Hill Tracts } \\ \text { CIA } & \text { Central Intelligence Agency } \\ \text { CIC } & \text { Counterintelligence Corps } \\ \text { CMC } & \text { Central Military Commission } \\ \text { CMPC } & \text { Central Military Party Committee } \\ \text { CPM } & \text { Communist Party of Malaya } \\ \text { CPP } & \text { Communist Party of the Philippines } \\ \text { CPT } & \text { Communist Party of Thailand } \\ \text { CRP } & \text { Central Reserve Police } \\ \text { CSC } & \text { Chief of Staff Committee } \\ & \end{array}$




$\begin{array}{ll}\text { CVF } & \text { Chosun Volunteer Forces } \\ \text { DCIC } & \text { Defence Coordination and Implementation Committee } \\ \text { DGFI } & \text { director-general of Forces Intelligence } \\ \text { DILG } & \text { Department of the Interior and Local Government } \\ \text { DNS } & \text { Department of National Security } \\ \text { DPC } & \text { Defense Planning Committee } \\ \text { DPP } & \text { Democratic Progressive Party } \\ \text { DPR } & \text { Dewan Perwakilan Rakyat } \\ \text { DPRD } & \text { Dewan Perwakilan Rakyat Daerah } \\ \text { DPRK } & \text { Democratic People's Republic of Korea } \\ \text { DRP } & \text { Democratic Republican Party } \\ \text { DRV } & \text { Democratic Republic of Vietnam } \\ \text { EDCOR } & \text { Armed Forces Economic Development Corps } \\ \text { EDSA } & \text { Epifanio de los Santos Avenue } \\ \text { FPI } & \text { Front Pembela Islam } \\ \text { FULRO } & \text { United Front for the Liberation of Oppressed Races } \\ \text { FWO } & \text { Frontier Works Organization } \\ \text { GAM } & \text { Gerakan Aceh Merdeka } \\ \text { GDP } & \text { gross domestic product } \\ \text { GNP } & \text { gross national product } \\ \text { GOC } & \text { general officer commanding } \\ \text { GPWD } & \text { General Political Warfare Department } \\ \text { GSH } & \text { General Staff Headquarters } \\ \text { HDO } & \text { Higher Defense Organization } \\ \text { IAF } & \text { Indian Air Force } \\ \text { IAS } & \text { Indian Administrative Service } \\ \text { IB } & \text { Intelligence Bureau } \\ \text { IBHI } & \text { Independent Bureau of Humanitarian Issues } \\ \text { IDF } & \text { Indigenous Defense Fighter } \\ \text { IISS } & \text { International Institute for Strategic Studies } \\ \text { IJI } & \text { Islamic Jamhoori Itihaad } \\ \text { IMET } & \text { International Military Education and Training } \\ \text { INA } & \text { Indian National Army } \\ \text { INC } & \text { Indian National Congress } \\ \text { INP } & \text { Integrated National Police } \\ \text { IPKF } & \text { Indian Peacekeeping Forces } \\ \text { IRBM } & \text { Intermediate Range Ballistic Missile } \\ \text { ISI } & \text { Inter Services Intelligence } \\ \text { ISOC } & \text { Internal Security Operations Command } \\ \text { ITBP } & \text { Indo-Tibetan Border Police } \\ \text { JCSC } & \text { Joint Chiefs of Staff Committee } \\ \text { JRB } & \text { Jatiyo Rakkhi Bahini } \\ \text { JUSMAG } & \text { Joint U.S. Military Advisory Group } \\ \text { JVP } & \text { Janatha Vimukthi Peramuna } \\ \text { KBL } & \text { Kilusang Bagong Lipunan } \\ \text { KCIA } & \text { Korean Central Intelligence Agency } \\ \text { KMT } & \text { Kuomintang } \\ & \end{array}$




$\begin{array}{ll}\text { KODAM } & \text { Regional Military Commands } \\ \text { KODIM } & \text { District Military Commands } \\ \text { Kopassus } & \text { Special Forces Command } \\ \text { Kopkamtib } & \text { Command for the Restoration of Security and Order } \\ \text { KORAML } & \text { Subdistrict Military Commands } \\ \text { KOREM } & \text { Resort Military Commands } \\ \text { Kostrad } & \text { Army Strategic Reserve Command } \\ \text { KPA } & \text { Korean People's Army; also North Korean People's Armed Forces } \\ \text { KWP } & \text { Korean Workers' Party } \\ \text { LDP } & \text { Liberal Democratic Party } \\ \text { LTTE } & \text { Liberation Tigers of Tamil Eelam } \\ \text { MAC } & \text { Mainland Affairs Council } \\ \text { MAF } & \text { Malaysian Armed Forces } \\ \text { MDPC } & \text { Ministry of Defense Party Committee } \\ \text { Metrocom } & \text { Metropolitan Command } \\ \text { MILF } & \text { Moro Islamic Liberation Front } \\ \text { MINDEF } & \text { Ministry of Defense } \\ \text { MND } & \text { Ministry of National Defense } \\ \text { MNF } & \text { multinational force } \\ \text { MNLF } & \text { Moro National Liberation Front } \\ \text { MoD } & \text { Ministry of Defence (India) } \\ \text { MOD } & \text { Ministry of Defense (Bangladesh) } \\ \text { MOEs } & \text { military-owned enterprises } \\ \text { MOFA } & \text { Ministry of Foreign Affairs } \\ \text { MoU } & \text { Memorandum of Understanding } \\ \text { MPAF } & \text { Ministry of People's Armed Forces } \\ \text { MPC } & \text { Military Party Committee } \\ \text { MPR } & \text { Majelis Permusyawaratan Rakyat } \\ \text { MPS } & \text { Military Planning Staff } \\ \text { MQM } & \text { Movement of the Mohajirs } \\ \text { MRD } & \text { Movement for the Restoration of Democracy } \\ \text { MTCs } & \text { military trial courts } \\ \text { MTDP } & \text { Mid-Term Defense Program } \\ \text { NAMFREL } & \text { National Movement for Free Elections } \\ \text { Napolcom } & \text { National Police Commission } \\ \text { NDC } & \text { National Defense Commission } \\ \text { NDPO } & \text { National Defense Program Outline } \\ \text { NDSC } & \text { National Defense Security Command } \\ \text { NGO } & \text { Nongovernmental Organization } \\ \text { NICA } & \text { National Intelligence Coordinating Authority } \\ \text { NISA } & \text { National Intelligence Security Agency } \\ \text { NKCP } & \text { North Kalimantan Communist Party } \\ \text { NLC } & \text { National Logistics Cell } \\ \text { NLD } & \text { National League for Democracy } \\ \text { NLL } & \text { Northern Limitation Line } \\ \text { NPA } & \text { New People's Army } \\ \text { NSB } & \text { National Security Bureau } \\ & \end{array}$




$\begin{array}{ll}\text { NSC } & \text { National Security Council } \\
\text { NSI } & \text { National Security Intelligence } \\
\text { NUF } & \text { National United Front } \\
\text { OPM } & \text { Organisasi Papula Merdeka } \\
\text { OSS } & \text { Office of Strategic Studies } \\
\text { PA } & \text { People's Alliance Party } \\
\text { PACC } & \text { Presidential Anti-Crime Commission } \\
\text { PAF } & \text { Pakistan Air Force (Chapter } 3) \\
\text { PAF } & \text { Philippine Air Force (Chapter 6) } \\
\text { PAP } & \text { People's Action Party } \\
\text { PARAKU } & \text { Pasukan Rakyat Kalimantan Utara } \\
\text { PBF } & \text { Patriotic Burmese Forces } \\
\text { PC } & \text { Philippine Constabulary } \\
\text { PCJSS } & \text { Parbattya Chattagram Janashonghoti Samity } \\
\text { PDI-P } & \text { Partai Demokrasi Indonesia-Perjuangan } \\
\text { PERISTA } & \text { Perkembangan Istimewa Angkatan Tentera } \\
\text { PGRS } & \text { Pasukan Gerila Rakyat Sarawak } \\
\text { PK } & \text { Pusan-Kyungnam } \\
\text { PKI } & \text { Partai Kommunis Indonesia } \\
\text { PKP } & \text { Partai Keadilan dan Persatuan } \\
\text { PLA } & \text { People's Liberation Army } \\
\text { PLOTE } & \text { People's Liberation Organization of Tamil Eelam } \\
\text { PMA } & \text { Philippine Military Academy } \\
\text { PNI } & \text { Partai Nasionalis Indonesia } \\
\text { PNP } & \text { Philippine National Police } \\
\text { PPP } & \text { Pakistan People's Party } \\
\text { PPP } & \text { Partai Persatuan Pembangunan } \\
\text { PPR } & \text { political party regulations } \\
\text { PRC } & \text { People's Republic of China } \\
\text { PSB } & \text { Presidential Security Battalion } \\
\text { PSC } & \text { Presidential Security Command } \\
\text { PSO } & \text { principal staff officer } \\
\text { PSU } & \text { Presidential Security Unit } \\
\text { PVL } & \text { Philippine Veterans Legion } \\
\text { RAM } & \text { Reform the Armed Forces of the Philippines Movement } \\
\text { RAW } & \text { Research and Analysis Wing } \\
\text { RMAF } & \text { Royal Malaysian Air Force } \\
\text { RMN } & \text { Royal Malaysian Navy } \\
\text { ROC } & \text { Republic of China on Taiwan } \\
\text { RPKAD } & \text { Army Para-Commando Regiment } \\
\text { RUCs } & \text { Regional Unified Commands } \\
\text { SAF } & \text { Singapore Armed Forces } \\
\text { SAS } & \text { Special Air Service } \\
\text { SCAP } & \text { supreme commander for the Allied Powers } \\
\text { SDC } & \text { Subcommittee on Defense Cooperation } \\
\text { SDF } & \text { Self-Defense Forces } \\
\text { SDP } & \text { Social Democratic Party } \\
& \end{array}$




$\begin{array}{ll}\text { SEATO } & \text { Southeast Asia Treaty Organization } \\ \text { SEC } & \text { Second Economy Commission } \\ \text { SLFP } & \text { Sri Lanka Freedom Party } \\ \text { SLORC } & \text { State Law and Order Restoration Council } \\ \text { SOEs } & \text { state-owned enterprises } \\ \text { SPDC } & \text { State Peace and Development Council } \\ \text { TADA } & \text { Terrorists and Disruptive Activities Act } \\ \text { TNA } & \text { Tamil National Army } \\ \text { TNI } & \text { Tentera Nasional Indonesia (Indonesian National Defense Forces) } \\ \text { UMEH } & \text { Union of Myanmar Economic Holdings, Ltd. } \\ \text { UMNO } & \text { United Malays National Organization } \\ \text { UNP } & \text { United National Party } \\ \text { UNTAET } & \text { United Nations Transitional Authority for East Timor } \\ \text { USAFFE } & \text { U.S. Armed Forces in the Far East } \\ \text { USAMGIK } & \text { American Military Government (in Korea) } \\ \text { USVA } & \text { U.S. Veterans Administration } \\ \text { VCP } & \text { Vietnamese Communist Party } \\ \text { VFA } & \text { Visiting Forces Agreement } \\ \text { VPA } & \text { Vietnamese People's Army }\end{array}$


